\title{
Singular arcs in the optimal control of a parabolic equation*
}

\author{
J. Frédéric Bonnans ${ }^{1}$
}

\begin{abstract}
We present a theory of singular arc, and the corresponding second order necessary and sufficient conditions, for the optimal control of a semilinear parabolic equation with scalar control applied on the r.h.s. We obtain in particular an extension of Kelley's condition, and the characterization of a quadratic growth property for a weak norm.
\end{abstract}

\section{INTRODUCTION}

We consider in this paper an optimal control problem of a parabolic equation with a scalar control subject to bounds, in which the state equation and integrand of cost function are affine functions of the control. Such affine control problems have been extensively studied in the ODE setting. Concerning second order optimality conditions which are the subject of the paper, in the totally singular case (the one when the bounds are never active), the key results are the extended Legendre condition due to Kelley [20], and the Goh transform [18] allowing to obtain second order necessary conditions involving the primitive of the control rather than the control itself. Dmitruk [15] derived sufficient conditions for weak optimality, and in [14], [16] obtained necessary or sufficient optimality conditions in the case of a non unique multiplier. In the singular-bang setting, Poggiolini and Stefani [25] obtained second-order sufficient conditions for the strong local optimality for the minimum time problem, and Aronna et al. [1] obtained second-order necessary conditions, and some sufficient conditions without uniqueness of the multiplier.

There are very few papers on the optimal control of PDEs for affine control systems. When the control is distributed, if the constraints are not active on some open subset, it is sometimes possible to give an explicit expression of the control: this is the theory of generalized bang-bang control, see Bergounioux and Tiba [3], Tröltzsch [26], Bonnans and Tiba [5].

In the elliptic case, Casas [10] considered the case of a distributed control and obtained second order sufficient conditions. While the technique is quite specific since time does not appear, these sufficients conditions are in the spirit of those obtained in Goh's theory, since they involve an Hilbert norm that is weaker than the $L^{2}$ norm of the control. Casas, Herzog and Wachsmuth [11] consider the case of an $L^{1}$ cost function, which may be viewed as an affine control problem if we take as new control the positive and negative parts of the original control. Casas, Clason and Kunisch

\footnotetext{
*Proc. 13th European Control Conference, July 17-19, Zurich, to appear. This work was supported by the ITN SADCO European network.

${ }^{1}$ INRIA-Saclay and Centre de Mathématiques Appliquées, Ecole Polytechnique, 91128 Palaiseau, France Frederic.Bonnans at inria.fr
}

[12] study related problems in the setting of a parabolic equation. Let us also mention the recent paper discussing several models [24].

Therefore there exists presently no analogous of the Goh theory in the setting of control of parabolic equations; our goal is to set some first steps in this direction. The paper is organized as follows. We present the problem, and derive the second order necessary conditions in Goh's form in section II. Then we derive the second order sufficient conditions, that characterize quadratic growth in a weak norm, in section III. Some numerical experiments, that give an experimental argument in favor of the existence of singular arcs for optimal control problems of parabolic equations, are presented in section IV. In section V, we make additional numerical experiments for the case when the control is constrained to be Lipschitz with constant 1 . We show that, as might be expected, a Fuller chattering phenomenon similar to the one described in [17] occurs. We conclude in section VI by discussing some open problems and possible extensions.

\section{Setting AND CLASSiCAl RESUlts}

\section{A. Setting}

Let $\Omega$ be a smooth open subset of $\mathbb{R}^{n}$, with $n \leq 3$. Set $Q:=\Omega \times(0, T)$. We recall that, for $\mu \in[1, \infty]$ and $p \in \mathbb{N}$, $p \geq 1, W^{p, \mu}(\Omega)$ denotes the Sobolev space of functions in $L^{\mu}(\Omega)$ with derivatives up to order $p$ (taken in the distribution sense) in $L^{\mu}(\Omega)$. We denote the closure of $\mathcal{D}(\Omega)$ (set of $C^{\infty}$ functions over $\Omega$ with compact support) in $W^{p, \mu}(\Omega)$ by $W_{0}^{p, \mu}(\Omega)$, and set $H^{p}(\Omega):=W^{p, 2}(\Omega)$ and $H_{0}^{p}(\Omega):=$ $W_{0}^{p, 2}(\Omega)$. Similarly, $W^{2,1, \mu}(Q)$ denotes the Sobolev space of functions in $L^{\mu}(Q)$ whose second derivatives in space and first derivative in time belong to $L^{\mu}(Q)$, and $H^{2,1}(Q):=$ $W^{2,1,2}(Q)$. Set also

$$
W_{\Sigma}^{2,1, \mu}(Q):=\left\{y \in W^{2,1, \mu}(Q) ; \quad y=0 \text { on } \partial \Omega \times(0, T)\right\} .
$$

The critical value

$$
\mu_{c}:=\frac{1}{2}(n+2)
$$

is such that, see [21, Rem. 2.5, p. 21]:

$$
W^{2,1, \mu}(Q) \subset L^{\infty}(Q) \text {, for all } \mu>\mu_{c} .
$$

Fix $\gamma \geq 0$ and $T>0$. We assume that

$$
\text { (i) } y_{0} \in W_{0}^{1, \infty}(\Omega) ; \quad \text { (ii) } B \in W_{0}^{2, \infty}(\Omega) \text {. }
$$

We consider the following semilinear parabolic controlled equation [21]:

$$
\left\{\begin{array}{l}
\dot{y}(x, t)-\Delta y(x, t)+\gamma y^{3}(x, t)=u(t) B(x) \\
\quad \text { in } Q:=\Omega \times[0, T], \\
y=0 \text { on } \partial \Omega \times[0, T] ; \quad y(\cdot, 0)=y_{0} .
\end{array}\right.
$$


This is a prototype of a semilinear equation; taking the explicit nonlinearity $y^{3}$ allows to simplify the analysis of the nonlinearity and to concentrate on the essential features. Indeed, in the case when $\gamma=0$, although the problem is convex, our characterization of quadratic growth is still of interest.

Consider the cost function

$$
J(u, y)=\frac{1}{2} \int_{Q}\left(y(x, t)-y_{d}(x, t)\right)^{2} \mathrm{~d} x \mathrm{~d} t+\alpha \int_{0}^{T} u(t) \mathrm{d} t .
$$

Here $\alpha \in \mathbb{R}$, the desired state $y_{d}$ belongs to $L^{\bar{\mu}}(Q)$, for some $\bar{\mu}$ such that

$$
\bar{\mu}>\mu_{c}=\frac{1}{2}(n+2) .
$$

We have control bounds

$$
a \leq u(t) \leq b, \quad \text { for all } t \in(0, T),
$$

for some real numbers $a<b$. The optimal control problem is therefore (we will be more precise on the functional framework later):

$$
\operatorname{Min}_{u, y} J(u, y) \text { s.t. (5) and (7). }
$$

The well-posedness of the state equation can be deduced from the following result, in which we consider a more general r.h.s.:

$$
\left\{\begin{array}{l}
\dot{y}(x, t)-\Delta y(x, t)+\gamma y^{3}(x, t)=f(x, t) \\
\quad \text { in } Q:=\Omega \times[0, T] \\
y=0 \text { on } \partial \Omega \times[0, T] ; \quad y(\cdot, 0)=y_{0} .
\end{array}\right.
$$

Proposition 2.1: Let $f \in L^{\infty}(Q)$ and $y_{0} \in W_{0}^{1, \infty}$. Then (8) has a unique solution $y[f]$ in $W^{2,1, q}(Q)$ for all $q \in$ $(1, \infty)$, and the mapping $f \mapsto y[f]$ is of class $C^{\infty}$ from $L^{\infty}(Q)$ into $W^{2,1, \mu}(Q)$.

Proof: See e.g. [6], [21].

A composition of $C^{\infty}$ mappings being of class $C^{\infty}$, we obtain that

Corollary 2.2: With each $u \in L^{\infty}(0, T)$ is associated a unique state $y[u] \in W^{2,1, q}(Q)$, for all $q \in[2, \infty)$, and the mapping $u \mapsto y[u]$ is of class $C^{\infty}$.

Theorem 2.3: The problem $(P)$ has a nonempty set of solutions. If $\gamma=0$, then the solution is unique.

Proof: The existence is obtained by standard arguments based on minimizing sequences, passing to the limit on the nonlinearity of the state equation, see e.g. [6], [21]. If $\gamma=0$ since the cost function is strictly convex w.r.t. the state, the optimal state is unique, and then so is the optimal control.

In the sequel we denote by $(\bar{u}, \bar{y})$ a solution of $(P)$.

\section{B. First order optimality system}

The costate equation is

$$
\left\{\begin{array}{l}
-\dot{\bar{p}}-\Delta \bar{p}+3 \gamma \bar{y}^{2} \bar{p}=\bar{y}-y_{d} \quad \text { in } Q \\
\bar{p}=0 \text { on } \partial \Omega \times[0, T] ; \quad \bar{p}(\cdot, T)=0 .
\end{array}\right.
$$

Since $y_{d} \in L^{\bar{\mu}}(Q)$, this equation has a unique solution $\bar{p} \in$ $W^{2,1, \bar{\mu}}(Q) \subset L^{\infty}(Q)$. We denote by $F(u):=J(u, y[u])$ the cost viewed as function of the control only. This is a function of class $C^{\infty}$ over $L^{\infty}(0, T)$. In the context of affine control problems, it is customary to call switching function the following amount

$$
\Psi(t):=\alpha+\int_{\Omega} B(x) \bar{p}(x, t) \mathrm{d} x .
$$

The linearized state equation is

$$
\left\{\begin{array}{l}
\dot{z}-\Delta z+3 \gamma \bar{y}^{2} z=v(t) B(x) \quad \text { in } Q, \\
z=0 \text { on } \partial \Omega \times[0, T] ; \quad z(\cdot, 0)=0 .
\end{array}\right.
$$

For $v \in L^{2}(Q)$, it has a unique solution $z[v] \in H^{2,1}(Q)$. The following result is classical.

Lemma 2.4: The switching function coincides with the derivative of $F$, in the sense that

$$
D F(\bar{u}) v=\int_{0}^{T} \Psi(t) v(t) \mathrm{d} t, \quad \text { for all } v \in L^{\infty}(0, T) .
$$

Proof: Let $z=z[v]$ denote the solution of the linearized state equation (11). By the chain rule, we have that using the integration by parts formula

$$
\begin{aligned}
D F(u) v & =\int_{Q}\left(y-y_{d}\right) z \mathrm{~d} x \mathrm{~d} t+\alpha \int_{0}^{T} v(t) \mathrm{d} t \\
& \left.=\int_{Q}\left(-\dot{\bar{p}}-\Delta \bar{p}+3 \gamma \bar{y}^{2} \bar{p}\right)\right) z(x, t) \mathrm{d} x \mathrm{~d} t \\
& =\quad+\alpha \int_{0}^{T} v(t) \mathrm{d} t \\
& =\int_{Q} \bar{p}\left(\dot{z}-\Delta z+3 \gamma \bar{y}^{2} z\right) \mathrm{d} x \mathrm{~d} t+\alpha \int_{0}^{T} v(t) \mathrm{d} t \\
& =\int_{Q} \bar{p}(x, t) B(x) v(t) \mathrm{d} x \mathrm{~d} t+\alpha \int_{0}^{T} v(t) \mathrm{d} t .
\end{aligned}
$$

The conclusion follows.

The (again classical) first order optimality conditions follow, denoting the contact set by $I(\bar{u})=I_{a}(\bar{u}) \cup I_{b}(\bar{u})$, where

$$
I_{a}(\bar{u}):=\{t \in(0, T) ; u(t)=a\}
$$

and

$$
I_{b}(\bar{u}):=\{t \in(0, T) ; \quad u(t)=b\} .
$$

Note that these sets are defined up to a null measure set.

Proposition 2.5: We have that up to a null measure set:

$$
\{t ; \Psi(t)>0\} \subset I_{a}(\bar{u}) ; \quad\{t ; \Psi(t)<0\} \subset I_{b}(\bar{u}) .
$$

We next need to analyze the first and second derivative of the switching function. The switching function has a derivative in $L^{2}(0, T)$, and since $B \in W_{0}^{2, \infty}(\Omega)$, we have that

$$
\left\{\begin{array}{l}
\dot{\Psi}(t):=-\int_{\Omega} B(x) \Delta \bar{p}(x, t) \mathrm{d} x \\
+\int_{\Omega} B(x)\left(3 \bar{y}^{2}(x, t) \bar{p}(x, t)-\bar{y}(x, t)+y_{d}(x, t)\right) \mathrm{d} x \\
=-\int_{\Omega} \bar{p}(x, t) \Delta B(x) \mathrm{d} x \\
+\int_{\Omega} B(x)\left(3 \bar{y}^{2}(x, t) \bar{p}(x, t)-\bar{y}(x, t)+y_{d}(x, t)\right) \mathrm{d} x
\end{array}\right.
$$


As expected from the theory of affine control problems in the case of ODEs, the derivative of the switching function does not depend on the control. Set

$$
\left\{\begin{aligned}
\kappa(x, t) & :=1-6 \gamma \bar{p}(x, t) \bar{y}(x, t), \quad(x, t) \in Q ; \\
R(t) & :=\int_{\Omega} \kappa(x, t) B(x)^{2} \mathrm{~d} x .
\end{aligned}\right.
$$

It is easily checked that $\kappa(x, t)$ and $R(t)$ are essentially bounded. By (17), if $y_{d}$ is smooth enough, $\Psi$ has a second derivative in $L^{2}(0, T)$. The latter is necessarily an affine function of the control, and it is easily checked that the following holds:

$$
R(t):=-\frac{\partial \ddot{\Psi}(t)}{\partial u} .
$$

In view of Kelley's result in [20], and of its extension to the case of control constraints in [1], we may expect that $R(t) \geq 0$ if the control constraints are not active near time $t$. We will indeed prove this result.

\section{Classical second order necessary conditions}

We will state second order necessary conditions based on the polyhedricity concept [19], [23]; see e.g., in an elliptic framework, [8, Section 6.3]. We first need to define boundary and singular arcs.

Definition 2.6: Let $t_{1}, t_{2}$ belong to $[0, T]$ with $t_{1}<t_{2}$. We say that $\left(t_{1}, t_{2}\right)$ is a singular arc if there exists $\varepsilon>0$ such that $\bar{u}(t) \in[a+\varepsilon, b-\varepsilon]$ for a.a. $t \in\left(t_{1}, t_{2}\right)$, a lower bound arc if $\bar{u}(t)=a$ for a.a. $t \in\left(t_{1}, t_{2}\right)$, and an upper bound arc if $\bar{u}(t)=b$ for a.a. $t \in\left(t_{1}, t_{2}\right)$. Lower and upper bound arcs are called boundary arcs.

We will consider only maximal arcs (that are not stricly included in another arc) and assume that

$\left\{\begin{array}{l}\text { There are finitely many maximal arcs, } \\ \text { ands the union of their closure is }[0, T]\end{array}\right.$

We call the arc starting at time 0 (finishing at time $T$ an initial (a final) arc. and the strict complementarity hypothesis, in the sense that

$\left\{\begin{array}{l}\Psi \text { has nonzero values over each boundary arc, } \\ \Psi(0) \neq 0 \text { in case of an initial boundary arc, } \\ \Psi(T) \neq 0 \text { in case of an final boundary arc. }\end{array}\right.$

Note that, $\Psi$ being a continuous function, the above definition makes sense. We may then redefine "punctually" the contact sets $I_{a}(\bar{u})$ and $I_{b}(\bar{u})$ as

$$
\left\{\begin{array}{l}
I_{a}(\bar{u})=\{t \in[0, T] ; \Psi(t)>0\} \\
I_{b}(\bar{u})=\{t \in[0, T] ; \Psi(t)<0\} .
\end{array}\right.
$$

Assuming strict complementarity, we have that the critical cone $C(\bar{u})$ and its extension $C_{2}(\bar{u})$ to $L^{2}(0, T)$ have the following expressions:

$$
\left\{\begin{array}{l}
C_{2}(\bar{u})=\left\{v \in L^{2}(0, T) ; \quad v(t)=0, t \in I(\bar{u})\right\} \\
C(\bar{u}):=C_{2}(\bar{u}) \cap L^{\infty}(0, T) .
\end{array}\right.
$$

We recall that the linearized state equation and its solution denoted by $z[v]$ were defined in (11). The following quadratic form (where $\kappa \in L^{\infty}(Q)$ has been defined in (18)) is obviously well-defined and continuous over $L^{2}(\Omega)$ :

$$
Q(v):=\int_{Q} \kappa(x, t) z(x, t)^{2} \mathrm{~d} t
$$

Theorem 2.7: We have that

$$
Q(v) \geq 0 \text { for all } v \in C_{2}(\bar{u}) .
$$

Proof: The technique is classical and similar to the one in [8, Section 6.3].

\section{Goh transform}

We next extend the Goh transform theory [18] to the present setting as follows. Define the "new control" $w(t)=$ $\int_{0}^{t} v(t) \mathrm{d} t$, and the "new linearized state" $\xi=z-w(t) B(x)$. The latter satisfies $\dot{\xi}=\dot{z}-u B=\Delta z-3 \bar{y}^{2} z$, and so is solution of

$$
\left\{\begin{array}{l}
\dot{\xi}(x, t)-\Delta \xi(x, t)+3 \bar{y}(x, t)^{2} \xi(x, t) \\
=w(t) \Delta B(x)-3 w(t) \bar{y}(x, t)^{2} B(x) \quad \text { in } \Omega . \\
\xi=0 \text { on } \partial \Omega \times[0, T] ; \quad \xi(\cdot, 0)=0 .
\end{array}\right.
$$

Since $\bar{y} \in L^{\infty}(Q)$, (4) implies that (26) has a unique solution $\xi[w] \in W^{2,1, s}(Q)$ whenever $w \in L^{s}(0, T)$, with $s \in[2, \infty)$.

In view of (20) the set of primitives of critical directions in with value 0 at time 0 is therefore

$$
P C(\bar{u})=\left\{w \in W^{1, \infty}(0, T) ; \dot{w}(t)=0, \quad t \in I(\bar{u})\right\} .
$$

Set

$$
\hat{Q}(w)=\int_{Q} \kappa(x, t)(\xi[w](x, t)+w(t) B(x))^{2} \mathrm{~d} t .
$$

Again this quadratic form is well defined and continuous over $H^{1}(0, T)$, and obviously $Q(v)=\hat{Q}(w)$. By (25) and the previous discussion we get

Lemma 2.8: We have that $\hat{Q}(w) \geq 0$, for all $w \in P C(\bar{u})$.

Next, observe that the closure in $L^{\overline{2}}$ of primitives of critical directions is

$$
P C_{2}(\bar{u})=\left\{\begin{array}{l}
w \in L^{2}(0, T) ; \dot{w}(t)=0, \quad t \in I(\bar{u}) ; \\
w=0 \text { on an initial boundary arc. }
\end{array}\right\},
$$

where we take the time derivative of $w$ in the distribution sense, on the relative interior of the set of times where the constraint is active. Then $\hat{Q}$ has a continuous extension over $L^{2}(0, T)$. With lemma 2.8 we deduce that

Lemma 2.9: We have that $\hat{Q}(w) \geq 0$, for all $w \in P C_{2}(\bar{u})$. Corollary 2.10: We have that

$$
R(t) \geq 0, \quad \text { for } t \notin I(\bar{u}) .
$$

Proof: Consider the problem of minimizing the quadratic form $\hat{Q}$ over the set

$$
P C_{2}^{\prime}(\bar{u})=\left\{w \in L^{2}(0, T) ; \quad w=0 \text { on } I(\bar{u})\right\} .
$$

Since $P C_{2}^{\prime}(\bar{u}) \subset P C_{2}(\bar{u})$, we have that $\bar{w}=0$ is solution of this problem. By the Legendre condition for this problem (itself a consequence of Pontryagin's principle [9]), we deduce that the conclusion holds.

Remark 2.11: As in the finite dimensional setting we have recovered the Kelley condition [20]: when the control constraint is not active, (19) is nonnegative. 


\section{SECOND ORDER SUFFICIENT CONDITIONS}

We first state the main result of this section and skip the proofs for reason of brevity, but note that it involves several ideas from [1]; see [4] for details.

Consider the following condition:

$$
\left\{\begin{array}{l}
\text { There exists } \rho>0 \text { such that } \\
\hat{Q}(w) \geq \rho\|w\|_{2}^{2}, \text { for all } w \in P C_{2}(\bar{u}) .
\end{array}\right.
$$

Theorem 3.1: Let (20)-(21) and (32) hold, and assume that there are no bang-bang junction. Then there exists $\rho^{\prime}>0$ such that, if $u$ is a feasible point of $(P)$, then setting $v:=u-\bar{u}$ and $w(t)=\int_{0}^{t} v(s) \mathrm{d} s$, we have that

$J(\bar{u}+v) \geq J(\bar{u})+\rho^{\prime}\|w\|_{2}^{2}, \quad$ if $\|v\|_{2}$ is small enough. (33)

The proof is based on the following estimate of the remainder of the second order Taylor expansion of the cost function.

Proposition 3.2: Let $\bar{u}+v$ be feasible, and set $w(t)=$ $\int_{0}^{t} v(s) \mathrm{d} s$. Then we have that:

$J(\bar{u}+v)=J(\bar{u})+\int_{Q} \Psi v+\frac{1}{2} \hat{Q}(w)+O\left(\|y-\bar{y}\|_{\infty}\|w\|_{2}^{2}\right)$.

Remark 3.3: This is to be compared to the classical expansion, consequence of the Implicit Function Theorem, taking $L^{\infty}(0, T)$ as control space, i.e.

$$
J(\bar{u}+v)=J(\bar{u})+\int_{Q} \Psi v+\frac{1}{2} \hat{Q}(w)+O\left(\|v\|_{\infty}^{3}\right) .
$$

\section{NUMERICAL EXPERIMENTS}

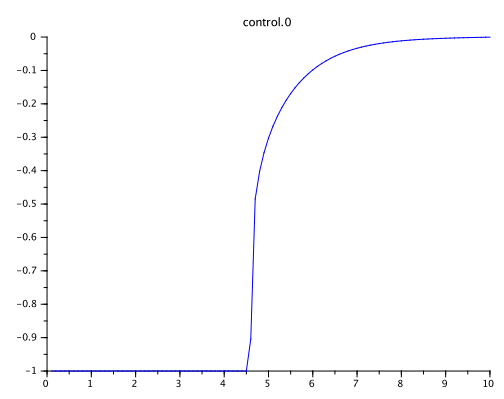

Fig. 1. Optimal control: $u \in[-1,1]$.

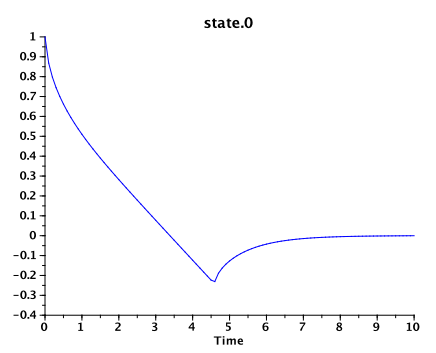

Fig. 2. State at boundary $x=0$.

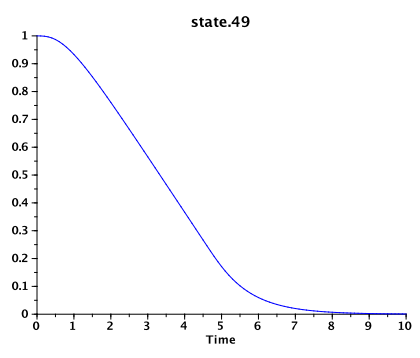

Fig. 3. State at boundary $x=1$.

An open question is the existence of singular arcs. We could consider the convex case when $\gamma=0$ and try to solve explicitly the optimality system; this however seems very difficult. We refer to Dhamo and Tröltzsch [13] for an example of an almost analytic resulution of an optimality sytem, in the context of parabolic equations.

On the other hand we can try numerical experiments and see if a singular arc seems to occur and is stable with respect to the discretization. We discretize the problem by standard finite differences, and solve the resulting optimal control problem with finitely many states using the optimal control toolbox BOCOP [7], which itself uses the nonlinear programming solver IPOPT [27].

The problem consists in controlling the one dimensional heat equation (and so here $\gamma=0$ ) by the Neumann condition at one end. More precisely, $\Omega=(0,1)$, the control is the Neumann condition at $x=0$, and the Neumann condition at $x=1$ is zero. We present the results with time horizon $T=10$, obtained with 50 space steps, 200 time steps, the implicit Euler scheme, and taking $y_{0}=1, y_{d}=0, \alpha=0$. We display next the optimal control, and the states function of time. The control constraint is $u(t) \in[-1,1]$, and we see that it is not active for $t \geq 5$. The same behavior is observed if we perturb the data and stepsize. So we may conjecture that this problem really has a singular arc.

\section{A FULLER TYPE PHENOMENON}

We next discuss the case when a constraint $\dot{u} \in[-1,1]$ is added to the previous problem. This can be interpreted as an infinite dimensional extension of the classical Fuller problem [17]. This is a case where the Goh transform should be performed two times in order to get a non degenerate quadratic form in the expression of the optimality conditions. The idea of performing multiple Goh transform was already in the original paper by Goh [18]. Another aspect of Fuller's problem is the chattering phenomenon: there is an infinite sequence of bang arcs (whose length decrease in a geometrical way) before the entry point for the singular arc.

So we may expect a similar behavior for the control of the heat equation. We display in figures 4 and 5 the Neumann condition and its derivative, with 500 time steps (such a large number is necessary in order to observe possible oscillations). We indeed observe damped oscillations that 
typically occur in the simulation of Fuller's problem, a zoom of which is displayed in figure 6 .

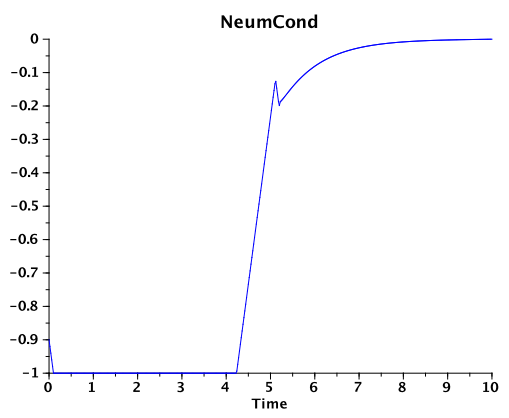

Fig. 4. Neumann condition $u \in[-1,1]$ with bounded derivative.

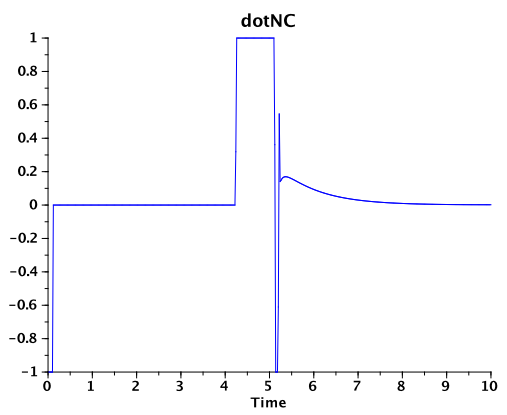

Fig. 5. Derivative of the Neumann condition, restricted to $[-1,1]$.

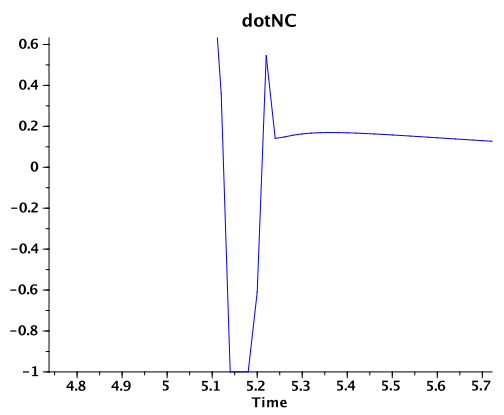

Fig. 6. Zoom on the derivative of the Neumann condition.

\section{CONCLUSION}

We have established second order necessary and sufficient conditions for the local optimality of an optimal control problem of a parabolic equation, the Hamiltonian being affine w.r.t. the control, in the case when the optimal control has a singular arc. The main result is a characterization of a weak form of quadratic growth, that extends to the setting of control of parabolic equations the recent results and techniques in [1] (see in particular the proof of our key theorem 3.1).
It is not easy to prove if such singular arcs occur. However, we give strong numerical arguments supporting such an existence, actually in the case of a boundary control.

Quite often, in real engineering devices, the action on the state equation is not the control itself, but rather the result of some integrations of the real control. Then the original control and $u$ becomes a state variable, which is of course subject to bounds. In the case of a single integration we have presented in the previous section some numerical results showing that a Fuller chattering phenomenon occurs. Observe that the former control now becomes a state. This raises the question of extending the present framework to the case of state constraints. Let us observe that little is known, even in the ODE setting; see however the analysis of optimality conditions in [22].

We note that, in the ODE setting, the design and proof of local convergence of shooting algorithms for such problems were recently obtained [2]. Since the backward heat equation is ill-posed, an extension of the shooting algorithm may be out of reach. However, similar algorithms based on junction times and on the final rather than initial value of the costate might be meaningful.

The present study deals with a simple case. It seems possible and of interest to extend our results in several directions. (i) The case of finitely many control variables, which does not seem to be essentially more complicated. (ii) The case when the coefficients of the control, in the cost function and state equation, also depend on the state, and when a terminal cost and finitely many final state constraints are present. While this case is well-understood in the ODE setting [1], the extension to the parabolic case clearly needs some efforts.

\section{REFERENCES}

[1] M.S. Aronna, J.F. Bonnans, A.V. Dmitruk, and P.A. Lotito. Quadratic order conditions for bang-singular extremals. Numerical Algebra, Control and Optimization, AIMS Journal, 2(3):511-546, 2012.

[2] S. Aronna, J.F. Bonnans, and P. Martinon. A well-posed shooting algorithm for optimal control problems with singular arcs. J. Optim. Theory Appl, (7763), 2011. Online First, Jan. 2013.

[3] Maïtine Bergounioux and Dan Tiba. General optimality conditions for constrained convex control problems. SIAM J. Control Optim., 34(2):698-711, 1996.

[4] J. F. Bonnans. Optimal control of a semilinear parabolic equation with singular arcs. Rapport de recherche RR-8099, INRIA, October 2012.

[5] J. F. Bonnans and D. Tiba. Control problems with mixed constraints and application to an optimal investment problem. Math. Rep. (Bucur.), 11(61)(4):293-306, 2009.

[6] J.F. Bonnans and P. Jaisson. Optimal control of a parabolic equation with time-dependent state constraints. SIAM J. Control Optim, 48(7):4550-4571, 2010.

[7] J.F. Bonnans, P. Martinon, and V. Grélard. Bocop v1.0.3: A collection of examples. url: www.bocop.org, June 2012.

[8] J.F. Bonnans and A. Shapiro. Perturbation analysis of optimization problems. Springer-Verlag, New York, 2000.

[9] E. Casas. Pontryagin's principle for state-constrained boundary control problems of semilinear parabolic equations. SIAM J. Control Optim., 35(4):1297-1327, 1997.

[10] E. Casas. Second order analysis for bang-bang control problems of PDE. SIAM J. Control Optim., 50(4):2355-2372, 2012.

[11] E. Casas, R. Herzog, and G. Wachsmuth. Optimality conditions and error analysis of semilinear elliptic control problems with $L^{1}$ cost functional. SIAM J. Optim., 22(3):795-820, 2012. 
[12] Eduardo Casas, Christian Clason, and Karl Kunisch. Parabolic control problems in measure spaces with sparse solutions. Technical report, Universität Graz, 2012.

[13] Vili Dhamo and Fredi Tröltzsch. Some aspects of reachability for parabolic boundary control problems with control constraints. Comput. Optim. Appl., 50(1):75-110, 2011.

[14] A. V. Dmitruk. Quadratic conditions for the Pontryagin minimum in an optimal control problem that is linear with respect to control, with a constraint on the control. Dokl. Akad. Nauk SSSR, 272(2):285-289, 1983.

[15] A.V. Dmitruk. Quadratic conditions for a weak minimum for singular regimes in optimal control problems. Soviet Math. Doklady, 18(2), 1977.

[16] A.V. Dmitruk. Quadratic order conditions for a Pontryagin minimum in an optimal control problem linear in the control. Math. USSR Izvestiya, 28:275-303, 1987.

[17] A.T. Fuller. Study of an optimum non-linear control system. J. of Electronics and Control, 15:63-71, 1963.

[18] B.S. Goh. Necessary conditions for singular extremals involving multiple control variables. J. SIAM Control, 4:716-731, 1966.

[19] A. Haraux. How to differentiate the projection on a convex set in Hilbert space. Some applications to variational inequalities. Journal Mathematical Society of Japan, 29:615-631, 1977.

[20] H.J. Kelley. A second variation test for singular extremals. AIAA Journal, 2:1380-1382, 1964.
[21] J.L. Lions. Contrôle des systèmes distribués singuliers. Dunod, Paris, 1983.

[22] H. Maurer. On optimal control problems with bounded state variables and control appearing linearly. SIAM J. Control Optimization, 15(3):345-362, 1977

[23] F. Mignot. Contrôle dans les inéquations variationnelles elliptiques. Journal of Functional Analysis, 22:130-185, 1976.

[24] H.J. Pesch, S. Bechmann, and J.-E. Wurst. Bang-bang and singular controls in optimal control problems with partial differential equations. In Conference for Decision and Control Proceedings, 2012. December 10-13, Maui, Hawaii, USA. Paper No. 0117.

[25] Laura Poggiolini and Gianna Stefani. Sufficient optimality conditions for a bang-singular extremal in the minimum time problem. Control Cybernet., 37(2):469-490, 2008.

[26] Fredi Tröltzsch. Regular Lagrange multipliers for control problems with mixed pointwise control-state constraints. SIAM J. Optim., 15(2):616-634 (electronic), 2004/05.

[27] Andreas Wächter and Lorenz T. Biegler. On the implementation of an interior-point filter line-search algorithm for large-scale nonlinear programming. Math. Program., 106(1, Ser. A):25-57, 2006. 